\title{
ClC-3 chloride channel protein induces G1 arrest in hepatocellular carcinoma Hep3B cells
}

\author{
RUI WANG ${ }^{1 *}$, BIJUN KANG $^{2 *}$, RENDONG HU ${ }^{2}$, YOUWAI HUANG ${ }^{2}$, ZIXI QIN ${ }^{2}$, JICHEN DU $^{3}$ and XI LIN ${ }^{2}$ \\ ${ }^{1}$ Beijing Key Laboratory of Magnetic Resonance Imaging Technology, Beijing 100191; ${ }^{2}$ Department of Pharmacology, \\ School of Medicine, Jinan University, Guangzhou, Guangdong 510632; ${ }^{3}$ Department of Neurology, \\ Aerospace Center Hospital, Peking University Aerospace Clinical College, \\ Beijing 100039, P.R. China
}

Received October 18, 2017; Accepted April 23, 2018

DOI: $10.3892 /$ or.2018.6416

\begin{abstract}
ClC-3 is a type of chloride channel that has multiple functions in tumorigenesis and tumor growth, and can be blocked by DIDS (4,4'-diisothiocyanostilbene-2,2'-disulfonic acid). In the present study, we found that DIDS inhibited the proliferation of Hep3B hepatocellular carcinoma (HCC) cells in a concentration-dependent manner. More in-depth research demonstrated that DIDS downregulated the protein expression levels of cyclin D1 and cyclin E, which are key proteins of the G1 phase. Additionally, we found that ClC-3 siRNA transfection induced $\mathrm{G} 1$ arrest in the Hep3B cells, confirming that ClC-3 is involved in the DIDS-induced inhibition of Hep3B cells. Moreover, the level of $\alpha$-fetoprotein (AFP), a negative prognostic indicator of $\mathrm{HCC}$, was decreased after treatment with DIDS and ClC-3 siRNA. In conclusion, we demonstrated that $\mathrm{ClC}-3$ can arrest the cell cycle at the $\mathrm{G} 1$ phase to inhibit cell proliferation, suggesting that $\mathrm{ClC}-3$ has the potential to be a novel target for HCC therapy and potentially improve the prognosis of HCC patients.
\end{abstract}

\section{Introduction}

Hepatocellular carcinoma (HCC) is a commonly diagnosed cancer, and its mortality rate was ranked second among all cancers worldwide in 2012 (1). However, therapy for HCC

Correspondence to: Dr Xi Lin, Department of Pharmacology, School of Medicine, Jinan University, Guangzhou, Guangdong 510632, P.R. China

E-mail: linx_jnu@163.com

Dr Jichen Du, Department of Neurology, Aerospace Center Hospital, Peking University Aerospace Clinical College, Beijing 100039, P.R. China

E-mail: djc721@163.com

*Contributed equally

Key words: cyclin D1, cyclin E, AFP, cell cycle, G1 arrest, Hep3B, DIDS, hepatocellular carcinoma remains unsatisfactory. Patients commonly experience relapse after surgery. Consequently, there is an urgent need to identify new targets for HCC therapy.

Cyclin D1 and cyclin E proteins are key factors that are involved in the transition from G1 phase to $S$ phase of the cell cycle. The aberrant expression of these proteins in G1 phase is a marker of malignancy $(2,3)$. It has been reported that, in breast cancer cells, the upregulation of cyclin D1 shortens G1 phase and promotes cell proliferation (4). In head and neck squamous cell carcinoma cells, protein kinase $\mathrm{C} \alpha$ and microRNAs can upregulate the expression of cyclin $\mathrm{E}$, resulting in oncogenic dysregulation (5). Metformin may induce G1 arrest by inhibiting E2F8 expression in lung cancer cells, which can lead to improvement in the overall survival of lung cancer patients (6).

DIDS (4,4'-diisothiocyanostilbene-2,2'-disulfonic acid) is a chloride channel blocker that has low specificity. It has been shown that DIDS can disrupt the glycolytic phenotype and decrease cell viability, and also enhance cell death and inhibit proliferation in colorectal cancer cells (7). In the human ovarian cancer cell line A2780, DIDS has been shown to inhibit cell adhesion and migration by regulatory volume decrease (RVD) and $\left[\mathrm{Ca}^{2+}\right]_{\mathrm{i}}$ changes (8). Furthermore, DIDS has demonstrated the ability to sensitize cells to chemotherapy and induce apoptotic cell death in resistant human glioblastoma multiforme cancer stem cells by cell swelling-induced cell cycle arrest after chemotherapy (9).

Chloride channels, one of the most important negative ion channels, regulate the cell cycle and volume (10-12), and the relationship between the volume-regulated regulated channels and cancer is an area of active research at present. $\mathrm{ClC}-3$, one of the families of volume-regulated channels, is involved in cell proliferation, apoptosis and migration (13). In human prostate cancer epithelial cells, the inhibition of $\mathrm{ClC}-3$ by an anti-ClC-3-specific antibody was found to decrease cell swelling which was activated by chloride currents (14). Some studies have shown that $\mathrm{ClC}-3$ promoted the resistance of cancer cells to chemotherapeutic drugs, such as cisplatin and etoposide (15-17). In osteosarcoma cells, ClC-3 induced G1 arrest by medicating cyclin D1 and cyclin E to modulate the proliferation and migration (18).

$\alpha$-Fetoprotein (AFP), the most commonly used tumor marker for the diagnosis of $\mathrm{HCC}$, is included in international 
guidelines for HCC surveillance (19-21). A high expression level of AFP is considered to be an indicator that a patient has HCC, and data show that AFP is elevated above the normal range in $70 \%$ of patients with HCC (22). AFP may be used for prediction and prognosis in HCC (23); when AFP levels decrease, this is considered to be a favorable prognostic indicator in HCC.

In the present study, we report a relationship between chloride channels and the cell cycle in HCC cells, as well as an association between chloride channels and AFP; to the best of our knowledge, this is the first report of such findings. In addition, the results suggested that $\mathrm{ClC}-3$ had an impact on the induction of $\mathrm{G} 1$ arrest and the levels of the prognostic indicator AFP. These results suggest that ClC-3 may be a novel potential candidate for the molecular-targeted treatment of HCC. Targeting ClC-3 may effectively kill cancer cells and potentially improve patient prognosis.

\section{Materials and methods}

Cell line. We acquired the human hepatocellular carcinoma Hep3B cell line and human L-02 hepatocytes from the American Type Culture Collection (ATCC; Manassas, VA, USA). We cultured cells in Dulbecco's modified Eagle's medium (DMEM) supplemented with $10 \%$ FBS in a humidified incubator containing $5 \% \mathrm{CO}_{2}$ at $37^{\circ} \mathrm{C}$. The medium was replaced and the cells were passaged twice per week to maintain logarithmic growth.

Reagent. DIDS was reconstituted in dimethyl sulfoxide (DMSO) (both from Sigma-Aldrich; Merck KGaA, Darmstadt, Germany).

DIDS treatment of Hep3B cells in vitro. In order to detect the function of DIDS by western blot analysis and flow cytometric analysis, $5 \times 10^{4}$ Hep3B cells were seeded into each well of 6 -well plates overnight. The cells were then treated with 0,50 , 100 or $200 \mu \mathrm{M}$ DIDS in DMEM with $2.5 \%$ FBS for $24 \mathrm{~h}$.

Western blot analysis. Hep3B cells were lysed in RIPA buffer with $1 \%$ Nonidet P-40 (20 mM Tris, $150 \mathrm{mM} \mathrm{NaCl}, 0.1 \mathrm{mM}$ EDTA). The amount of total proteins was quantified using the bicinchoninic acid (BCA) method. The proteins were separated according to molecular weight by SDS-PAGE and then a semidry transfer instrument (Bio-Rad Laboratories, Inc., Hercules, CA, USA) was used to transfer the proteins onto PVDF membranes. The membranes were blocked in 5\% skim milk, prior to incubation with primary and secondary antibodies (1:5,000; Sigma-Aldrich; Merck KGaA). Finally, the PVDF membranes were viewed with Molecular Analyst software (Bio-Rad Laboratories). The primary antibodies we used were as follows: anti-ClC-3 (1:1,000; cat. no. 13359; Cell Signaling Technology, Inc., Danvers, MA, USA), anti-cyclin D1 (1:1,000; cat. no. 2978; Cell Signaling Technology), anticyclin E (1:1,000; cat. no. 2273964; EMD Millipore, Billerica, MA, USA), anti-AFP (1:1,000; cat. no. 4448; Cell Signaling Technology), anti-GAPDH (1:5,000; cat. no. AP0060; Bioworld Technology, Inc., St. Louis Park, MN, USA) and anti- $\beta$-actin (1:5,000; cat. no. AP0063; Bioworld Technology).
Cell transfection. Following the manufacturer's protocol, a Silencer Small Interfering RNA (siRNA) Construction kit (Guangzhou RiboBio, Co., Ltd., Guangzhou, China) was used to synthesize dsRNA-ClC-3. Hep3B cells $\left(4 \times 10^{4}\right)$ were seeded into each well of a 6 -well plate overnight. Then in all of the wells, medium was replaced with DMEM containing $1 \%$ FBS and serum-free Opti-MEM I Medium (Gibco; Thermo Fisher Scientific, Inc., Waltham, MA, USA) (without antibiotics or fungicides), and the different treatments, including NC, RNA iMAX, siRNA-ClC-3-001, siRNA-ClC-3-002 and siRNA-ClC-3-003, were applied for $48 \mathrm{~h}$. There was no RNA sequence or no lipidosome used in the control group. There was Random sequence genome with lipidosome in the NC group. There was only lipidosome in RNA iMAX. There were ClC-3 siRNA sequences with lipidosome in the siRNA-ClC-3-002 or siRNA-ClC-3-003 group. Lipofectamine 2000 (Invitrogen; Thermo Fisher Scientific, Inc.) was used to transfect the siRNA $(10 \mathrm{nM})$ into the Hep3B cells. After 2 days, western blot analysis was conducted as described above. The sequences of $\mathrm{ClC}-3$ siRNA were: 5'-GAAGAGGUAU UGAAUGCUAdTdT-3'.

Quantitative real-time polymerase chain reaction ( $q P C R)$. Total RNA was extracted from the Hep3B cells with TRIzol reagent (Invitrogen; Thermo Fisher Scientific, Inc.) and reverse-transcribed into cDNA using oligo(dT) primers (Stratagene; Agilent Technologies, Inc., Santa Clara, CA, USA) and RevertAid Reverse Transcriptase (Thermo Fisher Scientific, Inc.). qPCR was performed with using an iCycler RT-PCR system (Bio-Rad Laboratories, Munich, Germany) and SuperReal PreMix SYBR Green (FP204-02; Tiangen Biotech, Co., Ltd., Beijing, China). We analyzed the data using the Pfaffli method. The primers for $\mathrm{ClC}-1$ to $\mathrm{ClC}-7$ were as follows: ClC-1 forward, 5'-CAG CAT CTG TGC TGC C-3' and reverse, 5'-GTG CTT AGC AAG AAA CTG GC-3'; ClC-2 forward, 5'-AGA CAA TCC CTA CAC CCT TCA A-3' and reverse, 5'-TGT CGG TAG AAC ACC TTG TCA C-3'; ClC-3 forward, 5'-CAA UGG AUU UCC UGU CAU ATT-3' and reverse, 5'-UAU GAC AGG AAA UCC AUU GTA-3'; ClC-4 forward, 5'-GCG TCT CAT CGG GTT TGC-3' and reverse, 5'-TTG CTC ACA ATG CCC TCT TTG-3'; ClC-5 forward, 5'-CTG TGC CAC TGC TTC AAC-3' and reverse, 5'-CTG AGG GCA AAT CCC ACT AA-3'; ClC-6 forward, 5'-GTC GCG CAA GAC TGT AAC CA-3' and reverse, 5'-CGG CGA AAT TCC ATA CCT G-3'; ClC-7 forward, 5'-CCC ACA CAA CGA GAA GCT CC-3' and reverse, 5'-ACT TGT CGA TAT TGC CCT TGA TG-3'. The primer sequences for GAPDH are were 5'-CTC ATG ACC ACA GTC CAT GC-3' (forward) and 5'-CAC ATT GGG GGT AGG AAC AC-3' (reverse).

Flow cytometric analysis of cell cycle distribution. Hep3B cells were treated with DIDS for $24 \mathrm{~h}$ or transfected with ClC-3 siRNA for $48 \mathrm{~h}$ and then centrifuged at 1,000 $\mathrm{x}$ g for $5 \mathrm{~min}$. Using phosphate-buffered saline (PBS), Hep3B cells were washed three times and resuspended in $75 \%$ alcohol overnight. The samples were stained by Triton-X 100 (Amresco, LLC, $\mathrm{OH}$, USA), $1 \mathrm{mg} / \mathrm{ml}$ propidium iodide (PI; Thermo Fisher Scientific, Inc.), DNase-free RNase (Thermo Fisher Scientific, Inc.) and PBS in dark for 1 or $2 \mathrm{~h}$. After staining the samples, we detected the cell cycle distribution with a flow cytometer (FACSCalibur; BD Biosciences, Franklin Lakes, NJ, USA). 
Flow cytometric analysis of apoptosis. Hep3B cells were treated with DIDS for $24 \mathrm{~h}$ and then centrifuged at 1,000 x g for $5 \mathrm{~min}$. The Hep3B cells were washed three times in PBS and stained with propidium iodide (PI) and Annexin V-FITC. After washing the samples and re-suspending them in binding buffer, flow cytometry (FACSCalibur; BD Biosciences) was used to assess the percentage of apoptotic cells. Fluorescence was detected at an excitation wavelength of $488 \mathrm{~nm}$ and an emission wavelength of $530 \mathrm{~nm}$ for Annexin V-FITC binding (as fluorescence channel FL1), and $488 \mathrm{~nm}$ excitation and red emission for PI (as fluorescence channel FL2).

MTT assay. For the MTT assay, $1 \times 10^{4}$ Hep3B cells or L-02 cells were seeded in each well of a 24-well plate overnight. Hep3B and L-02 cells were then treated with 0, 25, 50, 100, 200 and $400 \mu \mathrm{M}$ DIDS in DMEM with $2.5 \%$ FBS for $24 \mathrm{~h}$. Next, $50 \mu$ l MTT (3-(4,5-dimethyl-2-thiazolyl)-2,5-diphenyl2-H-tetrazolium bromide) solution was added to each well, and the plates were incubated for $3 \mathrm{~h}$ at $37^{\circ} \mathrm{C}$. The supernatant was then discarded and $1 \mathrm{ml}$ DMSO was added to each well. The absorbance was read at $570 \mathrm{~nm}$ using an automatic enzyme-linked immune detector (Bio-Rad Laboratories).

Statistical analysis. We analyzed all data by two-way ANOVA or independent-samples t-tests as appropriate. The data are presented as the mean \pm SEM. Statistical analyses of all quantitative data were performed with GraphPad software (GraphPad Software, Inc., La Jolla, CA, USA).

\section{Results}

Viability of Hep3B cells following treatment with different concentrations of DIDS. The effects of different concentrations of DIDS on Hep3B and L-02 cells are shown in Fig. 1. We treated Hep3B and L-02 cells with 0, 25, 50, 100, 200 and $400 \mu \mathrm{M}$ DIDS for $24 \mathrm{~h}$. With increasing concentrations, the viability of the Hep3B cells was decreased, whereas the viability of the L-02 cells remained almost constant. This indicated that DIDS has a strong effect on HCC Hep3B cells, but had almost no effect on L-02 cells (a normal liver cell line). Based on the results shown in Fig. 1, we determined the most effective and suitable concentrations for use in the following studies.

DIDS inhibits the proliferation of Hep3B cells by G1 arrest. In order to elucidate how DIDS affects the viability of Hep3B cells, we treated Hep3B cells with 0, 50, 100 and $200 \mu \mathrm{M}$ DIDS for $24 \mathrm{~h}$ prior to flow cytometry. It was demonstrated that DIDS inhibited the proliferation of cells by arresting cells in the G1 phase, preventing their transition into $S$ phase. With increasing concentrations of DIDS, the degree of inhibition was more obvious. At the same time, the results suggested that the distribution of cells was decreased in other phases of the cell cycle with increasing DIDS concentrations (Fig. 2A and B).

When apoptosis was analyzed by flow cytometry, it was demonstrated that DIDS had less influence on the apoptosis of Hep3B cells (Fig. 2C and D). Therefore, DIDS predominantly affected the proliferation of Hep3B cells by regulating cell cycle progression, but not through apoptosis.

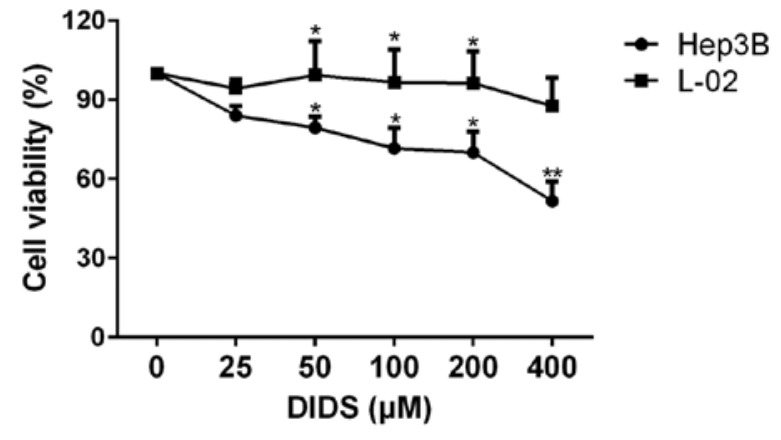

Figure 1. Viability of the Hep3B cells after treatment with different concentrations of DIDS. The hepatocellular carcinoma cell line Hep3B and normal liver cell line L-02 were treated with various concentrations of DIDS for $24 \mathrm{~h}$ and assessed by MTT assay. ${ }^{*} \mathrm{P}<0.05 ;{ }^{* *} \mathrm{P}<0.01$ (vs. Control, $\mathrm{n}=3$ ). DIDS, 4,4'-diisothiocyanostilbene-2,2'-disulfonic acid.

DIDS downregulates the proteins associated with G0/ G1 phase and AFP in Hep $3 B$ cells. To observe the mechanism by which DIDS induces G1 arrest in Hep3B cells, we detected the levels of the proteins responsible for G1 to $S$ phase transition by western blot analysis. The results indicated that, with the ascending concentrations of DIDS, the protein levels of cyclin D1 and cyclin E were gradually diminished in the Hep3B cells (Fig. 3A and B). Cyclin D1 and cyclin E, the main G1-associated proteins, are involved in driving the transition from G1 to S phase of the cell cycle. Unexpectedly, we noted that DIDS also inhibited the expression of AFP protein (Fig. 3A and B). The results indicated that DIDS may downregulate the protein levels of cyclin D1 and cyclin E and inhibit the protein expression of AFP (a biomarker of Hep3B cells), resulting in the inhibition of proliferation of Нер3В cells.

ClC-3 may serve a main role in Gl arrest induced by DIDS. We detected the relative mRNA expression levels of the chloride channel family, ClC-1 to ClC-7, in Hep3B cells by qPCR . The results revealed that, in contrast to the mRNA levels of ClC-2 and $\mathrm{ClC}-1$, the mRNA levels of ClC-3 were distinctly much higher (Fig. 4A).

Subsequently we detected the expression levels of $\mathrm{ClC}-3$ by western blotting in Hep3B cells treated with $0,50,100$ and $200 \mu \mathrm{M}$ DIDS for $24 \mathrm{~h}$, in order to confirm whether DIDS could block ClC-3 in Hep3B cells. As expected, the protein levels of ClC-3 were inhibited by DIDS treatment, with a higher concentration of DIDS resulting in more marked inhibition (Fig. 4B and C). These results suggest that $\mathrm{ClC}-3$, one member of the chloride channel family, may play a key role in G1 arrest and in inhibiting the protein expression of AFP in response to DIDS treatment.

ClC-3 siRNA transfection induces G1 arrest and AFP protein in Hep3B cells. In order to confirm whether $\mathrm{ClC}-3$ is involved in inducing G1 arrest and inhibiting the protein expression of AFP in Hep3B cells, we used transfection to silence the expression of $\mathrm{ClC}-3$ in Hep3B cells. First, we selected the most effective ClC-3 siRNA from si-ClC-3-001, si-ClC-3-002 and si-ClC-3-003 by detecting the expression levels of $\mathrm{ClC}-3$ protein through western blot analysis after transfection. The 
A
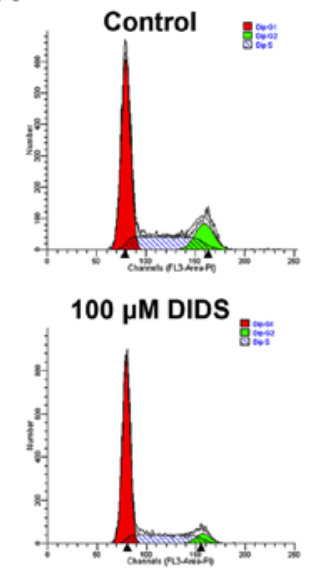

C
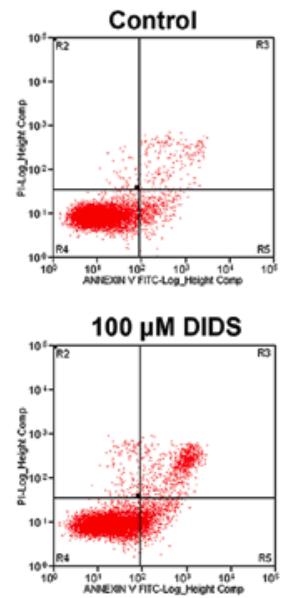

B

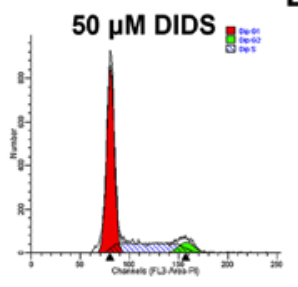

$200 \mu \mathrm{M}$ DIDS

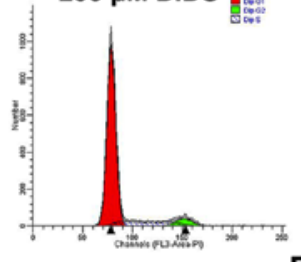

D
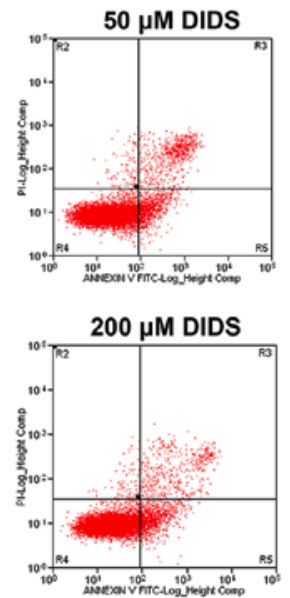
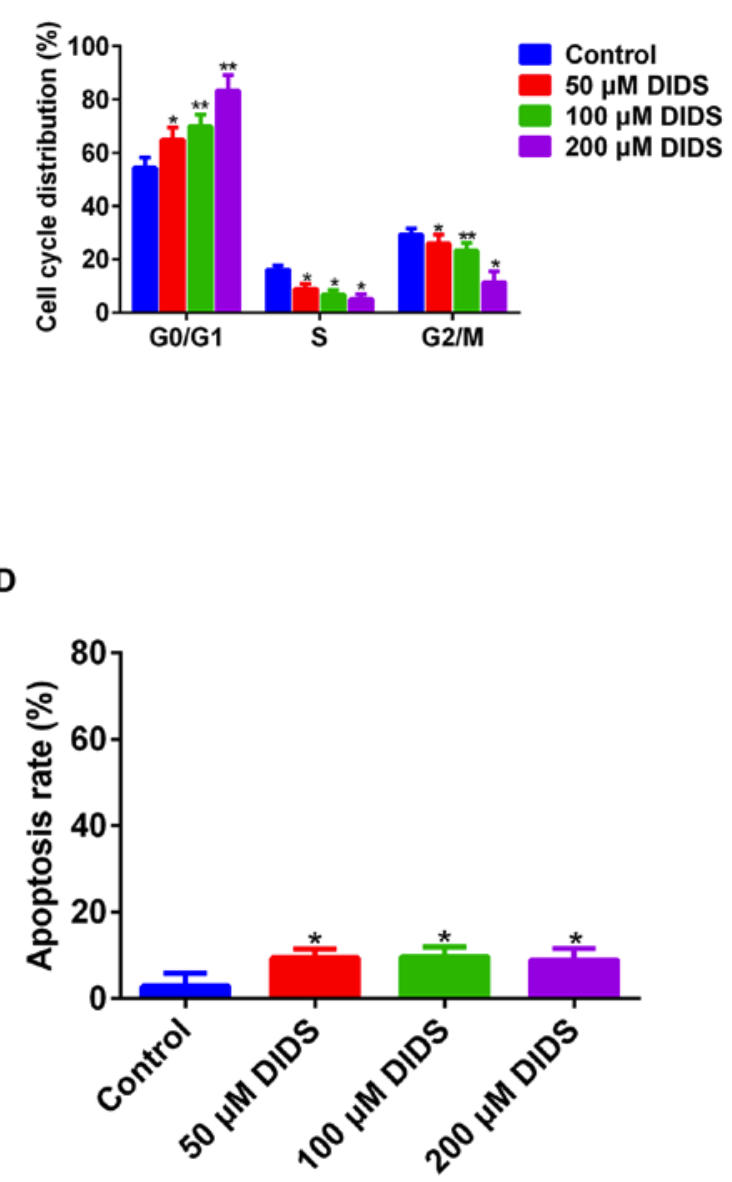

Figure 2. DIDS affects the proliferation of Hep3B cells by G1 arrest. (A and B) Cell cycle distribution was analyzed by flow cytometry in Hep3B cells treated with various concentrations of DIDS for $24 \mathrm{~h} .{ }^{*} \mathrm{P}<0.05 ;{ }^{* *} \mathrm{P}<0.01$ (vs. Control, $\mathrm{n}=3$ ). (C and D) Cell apoptosis was detected by Annexin V-FITC/PI staining and flow cytometry in Hep3B cells treated with various concentrations of DIDS for $24 \mathrm{~h}$. "P<0.05 (vs. Control, n=3). DIDS, 4,4'-diisothiocyanostilbene-2,2'-disulfonic acid.
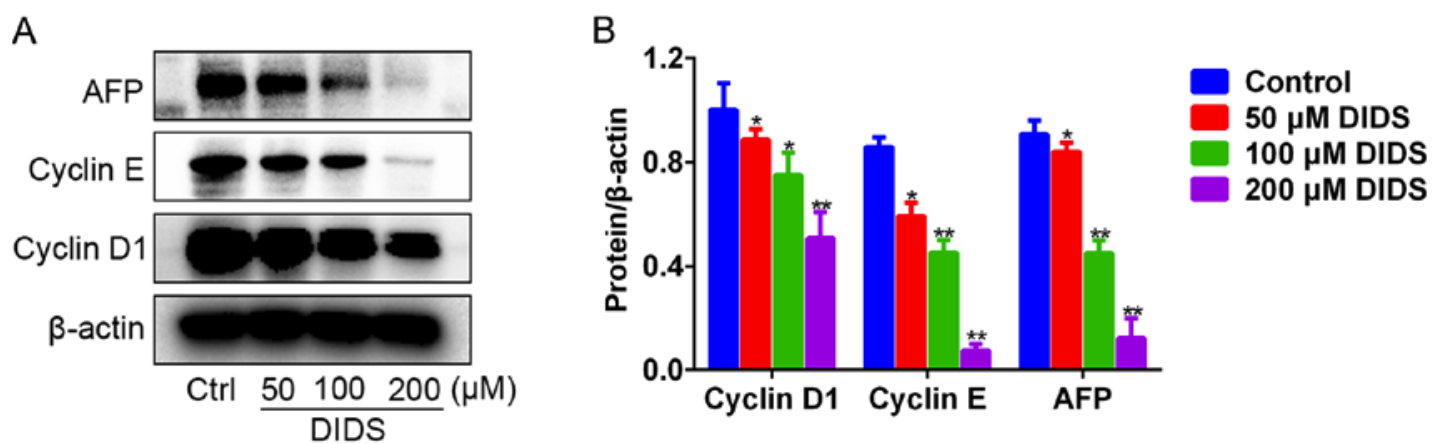

Figure 3. DIDS downregulates proteins associated with G0/G1 phase and $\alpha$-fetoprotein (AFP) in Hep3B cells. (A and B) The protein levels of cyclin E, cyclin D1 and AFP were detected by western blotting in the Hep3B cells treated with increasing concentrations of DIDS for $24 \mathrm{~h} .{ }^{*} \mathrm{P}<0.05 ;{ }^{* *} \mathrm{P}<0.01$ (vs. Control, $\mathrm{n}=3$ ). DIDS, 4,4'-diisothiocyanostilbene-2,2'-disulfonic acid.

silencing effects of si-ClC-3-001 and si-ClC-3-003 were found to be markedly greater (Fig. 5A and B).

Next, we detected the cell cycle distribution following transfection using flow cytometry. After silencing ClC-3, cell cycle progression was arrested at G1 phase and transition into $\mathrm{S}$ phase was suppressed (Fig. 5C and D). Finally, we confirmed that the levels of G1 phase-related proteins after the silencing of $\mathrm{ClC}-3$ were the same as those after
DIDS treatment. Cyclin D1 and cyclin E protein were both decreased in Hep3B cells treated with si-ClC-3-001 and si-ClC-3-003 (Fig. 5E and F). Simultaneously, the expression of AFP protein was also decreased (Fig. 5G and H). These results are consistent with the results obtained in DIDStreated cells, indicating that DIDS targets $\mathrm{ClC}-3$ to induce G1 arrest and to inhibit the expression of AFP in Hep3B cells, as predicted. 


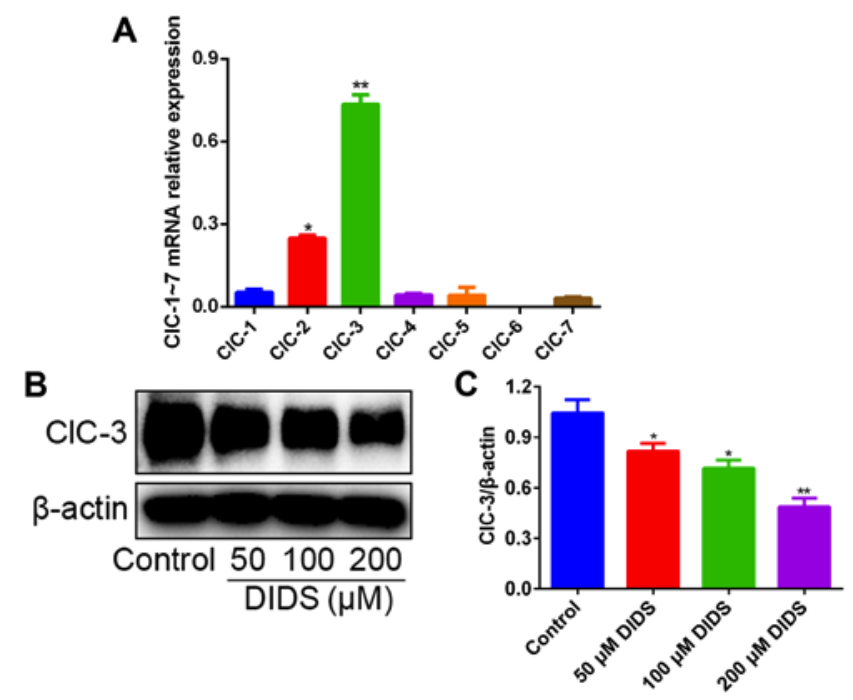

Figure 4. ClC-3 plays an important role in DIDS-induced G1 arrest. (A) Relative mRNA levels of different chloride channels were detected in Hep3B cells by RT-qPCR. "P $<0.05 ;{ }^{* *} \mathrm{P}<0.01$ (vs. Control, $\mathrm{n}=3$ ). ( $\mathrm{B}$ and $\mathrm{C}$ ) The protein levels of $\mathrm{ClC}-3$ were detected by western blotting in Hep3B cells treated with various concentrations of DIDS for $24 \mathrm{~h}$. ${ }^{*} \mathrm{P}<0.05 ;{ }^{* *} \mathrm{P}<0.01$ (vs. Control, $\mathrm{n}=3$ ). DIDS, 4,4'-diisothiocyanostilbene-2,2'-disulfonic acid.

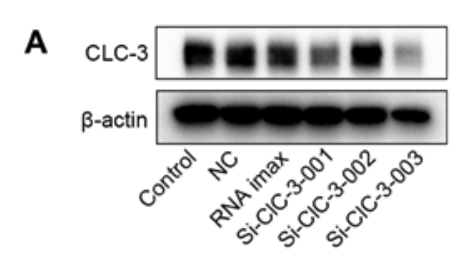

C

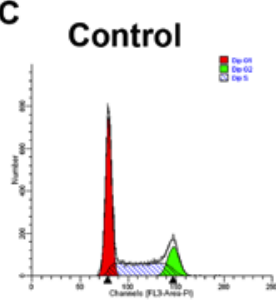

Si-CIC-3-001

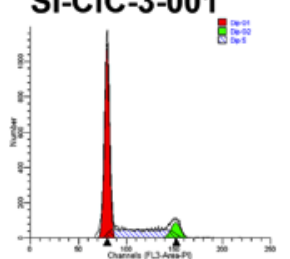

E

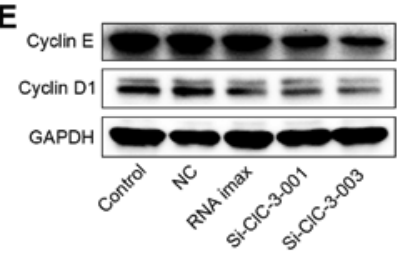

G

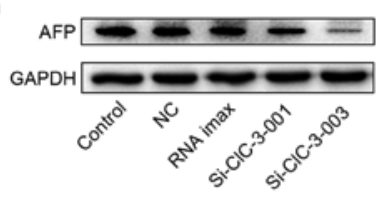

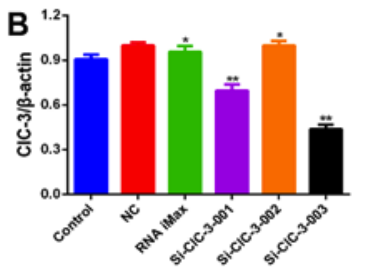

RNA iMax

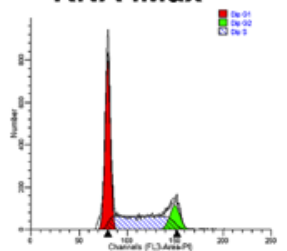

Si-CIC-3-003 D
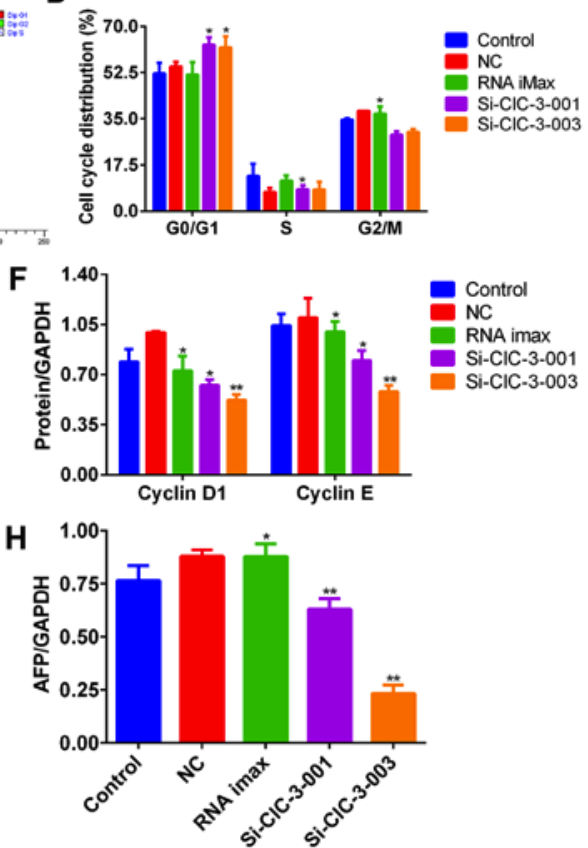

Figure 5. ClC-3-specific siRNA transfection induces G1 arrest and decreases the malignant potential of Hep3B cells. (A and B) The protein expression of ClC-3 was detected by western blot analysis in Hep3B cells treated with $\mathrm{ClC}-3$ siRNA. ${ }^{*} \mathrm{P}<0.05 ;{ }^{* *} \mathrm{P}<0.01$ (vs. Control, $\mathrm{n}=3$ ). (C and $\left.\mathrm{D}\right) \mathrm{Cell}$ cycle distribution was analyzed by flow cytometry in Hep3B cells treated with siRNA-ClC-3-001 and siRNA-ClC-3-003. ${ }^{*} \mathrm{P}<0.05 ;{ }^{* *} \mathrm{P}<0.01$ (vs. NC, $\mathrm{n}=3$ ). $(\mathrm{E}-\mathrm{H}) \mathrm{The}$ protein levels of cyclin E, cyclin D1 and AFP were detected by western blot analysis in Hep3B cells treated with siRNA-ClC-3-001 and siRNA-ClC-3-003. ${ }^{*}<0.05 ;{ }^{* *} \mathrm{P}<0.01$ (vs. NC, $n=3$ ). AFP, $\alpha$-fetoprotein; NC, negative control. 


\section{Discussion}

Ion channels are potential novel targets for improving the treatment of cancer. Chloride channels, including $\mathrm{ClC}-3$, are the most important negative ion channels. $\mathrm{ClC}-3$ plays a significant role in regulating the cell cycle, and can result in G1 arrest in nasopharyngeal carcinoma and osteosarcoma cells $(18,24)$. However, to the best of our knowledge, there has been no report of similar findings in HCC cells to date. Consequently, we researched $\mathrm{ClC}-3$ protein and the cell cycle in Hep3B cells (a HCC cell line), and found associations among $\mathrm{ClC}-3$, the cell cycle and the level of AFP.

First, we demonstrated that DIDS had a concentrationdependent effect on Hep3B cells, but had hardly any effect on L-02 cells. This supported our hypothesis that DIDS can inhibit the proliferation of HCC cells effectively, and also has little disadvantage to normal liver cells. It was reported that 100 and $250 \mu \mathrm{M}$ DIDS had no effect on normal liver cells at day 2 following treatment (25), which was consistent with our results that 25-200 $\mu \mathrm{M}$ DIDS had no effect on L-02 cells at $24 \mathrm{~h}$ (Fig. 1). This is a huge benefit in cancer therapy, as such a treatment could target HCC while having a limited effect on normal liver cells. Second, in order to determine how DIDS affects the proliferation of Hep3B cells, we used flow cytometry to detect the cell cycle distribution and cell apoptosis rate. It was observed that DIDS inhibited the proliferation of Hep3B cells through G1 arrest but not by promoting apoptosis. These results are consistent with previous research in other tumor cells $(18,24)$. Then, we detected the expression of the main proteins associated with G1 phase by western blot analysis, in order to demonstrate the mechanism of cell cycle regulation by DIDS. The data suggested that DIDS induced G1 arrest by downregulating the protein expression levels of cyclin D1 and cyclin E. Meanwhile, we observed that DIDS also reduced the protein level of AFP, which indicates that it may be able to improve the prognosis of $\mathrm{HCC}$ in patients.

However, DIDS is a broad-spectrum and non-specific blocker of volume-regulated chloride channels. In order to determine which volume-regulated chloride channels were affected, we performed qPCR to detect the expression levels of the chloride channel protein family in Hep3B cells. The mRNA expression of $\mathrm{ClC}-3$ was found to be the highest, while the second highest was $\mathrm{ClC}-2$ in Hep3B cells. The results of the western blotting suggested that the expression of $\mathrm{ClC}-3$ protein was obviously decreased with increasing concentrations of DIDS. Next, we silenced the expression of $\mathrm{ClC}-3$ specifically to confirm this hypothesis. The results showed that, when the protein expression of $\mathrm{ClC}-3$ was silenced, G1 arrest occurred and the protein levels of cyclin D1 and cyclin E were decreased. Meanwhile, the expression of AFP protein was markedly decreased following ClC-3 siRNA transfection. We believe that the siRNA sequences may have influence on Hep3B cells so that in NC (negative control), AFP expression was increased. Therefore, we compared the results of siRNA-ClC-3 with NC but Control to attenuate the influence of interference sequences in Hep3B cells. These results were consistent with the results obtained after treatment of the cells with DIDS.

As has been proposed in certain previous reports, $\mathrm{ClC}-3$, a volume-activated chloride channel, is an important regulator at the plasma membrane (24). DIDS may inhibit the protein levels of $\mathrm{ClC}-3$ at the plasma membrane to downregulate the expression of cyclin D1 and cyclin E, causing G1 arrest, potentially resulting in the improvement of prognosis. In this way, DIDS could prevent the growth of HCC cells and effectively kill them, while having a very limited effect on the growth of normal liver cells.

To the best of our knowledge, we are the first to report that chloride channels are associated with the HCC marker AFP. It has been reported that anti-AFP single-chain variable fragments can induce growth inhibition through the induction of G1 arrest and apoptosis in AFP-expressing HCC cell lines (26). Silencing of AFP was shown to induce G1 arrest in the HCC cell line EGHC-9901 (27). Therefore, it is possible that $\mathrm{ClC}-3$ could mediate AFP to induce $\mathrm{G} 1$ arrest in Hep3B cells. We will continue researching the mechanism of how ClC-3 mediates AFP and how AFP induces G1 arrest in HCC cells in our further studies, through siRNA transfection, co-immunoprecipitation assay and western blot analysis.

Blocking chloride channels can lead to the inhibition of cell proliferation and the arrest of cell cycle progression in human laryngeal cancer cells (28). It was reported that $\mathrm{ClC}-3$, the inhibition of which was shown to decrease the aggressiveness of neuroglioma cells by inhibiting the NF- $\mathrm{B}$ pathway, may be a novel therapeutic target and prognostic biomarker in neuroglioma (29). ClC-3 is a potential target for cancer therapy, and we found that it was highly expressed in Hep3B cells. This result supported our hypothesis that $\mathrm{ClC}-3$ may be an extremely valuable biotherapeutic target in HCC. Our research mainly focused on $\mathrm{ClC}-3$ protein, and we did not perform an in-depth study on the change of the $\mathrm{Cl}^{-}$current. Other mechanisms may also be responsible for our findings. We will conduct research on the $\mathrm{Cl}^{-}$current in our subsequent study. For further studies, we will continue our experiments using more HCC cell lines, such as HepG2 and Huh-7 cells, and we will demonstrate our findings in vivo.

\section{Acknowledgements}

We thank Dr Xi Lin (Department of Pharmacology, Medical College, Jinan University, Guangzhou; Department of Key Laboratory for Environmental Exposure and Health, Environment College, Jinan University, Guangzhou, China) and Dr Jichen Du (Department of Neurology, Aerospace Center Hospital, Peking University Aerospace Clinical College, Beijing, China) for the helpful discussion.

\section{Funding}

The present study was funded by the Science and Technology Planning Project of Guangdong Province(no.2014A020211022), and the Guangdong Province Ordinary University Innovation Team Project and Science and Technology Planning Project of Guangzhou Canton (no. 201510010074).

\section{Availability of data and materials}

The datasets used during the present study are available from the corresponding author upon reasonable request. 


\section{Authors' contributions}

LX, DJ and WR and conceived and designed the study. WR, $\mathrm{KB}, \mathrm{HR}$ and $\mathrm{HY}$ performed the experiments. WR and KB wrote the paper. HR and QZ reviewed and edited the manuscript. All authors read and approved the manuscript and agree to be accountable for all aspects of the research in ensuring that the accuracy or integrity of any part of the work are appropriately investigated and resolved.

\section{Ethics approval and consent to participate}

The study was conducted using cell lines and no human tissues were used. Thus no ethical approval and patient consent were required.

\section{Consent for publication}

Not applicable.

\section{Competing interests}

The authors state that they have no competing interests.

\section{References}

1. Zhang Y, Ren JS, Shi JF, Li N, Wang YT, Qu C, Zhang Y and Dai M: International trends in primary liver cancer incidence from 1973 to 2007. BMC Cancer 15: 94, 2015.

2. Park GH, Song HM and Jeong JB: The coffee diterpene kahweol suppresses the cell proliferation by inducing cyclin D1 proteasomal degradation via ERK1/2, JNK and GKS3 $\beta$-dependent threonine-286 phosphorylation in human colorectal cancer cells. Food Chem Toxicol 95: 142-148, 2016.

3. Yuan C, Zhu X, Han Y, Song C, Liu C, Lu S, Zhang M, Yu F, Peng Z and Zhou C: Elevated HOXA1 expression correlates with accelerated tumor cell proliferation and poor prognosis in gastric cancer partly via cyclin D1. J Exp Clin Cancer Res 35: 15, 2016.

4. Quelle DE, Ashmun RA, Shurtleff SA, Kato JY, Bar-Sagi D, Roussel MF and Sherr CJ: Overexpression of mouse D-type cyclins accelerates G1 phase in rodent fibroblasts. Genes Dev 7 : $1559-1571,1993$

5. Cohen EE, Zhu H, Lingen MW, Martin LE, Kuo WL, Choi EA, Kocherginsky M, Parker JS, Chung $\mathrm{CH}$ and Rosner MR: A feed-forward loop involving protein kinase Calpha and microRNAs regulates tumor cell cycle. Cancer Res 69: 65-74, 2009.

6. Jin DH, Kim Y, Lee BB, Han J, Kim HK, Shim YM and Kim DH: Metformin induces cell cycle arrest at the G1 phase through E2F8 suppression in lung cancer cells. Oncotarget 8: 101509-101519, 2017.

7. Amorim R, Pinheiro C, Miranda-Gonçalves V, Pereira H, Moyer MP, Preto A and Baltazar F: Monocarboxylate transport inhibition potentiates the cytotoxic effect of 5-fluorouracil in colorectal cancer cells. Cancer Lett 365: 68-78, 2015

8. Li M, Wang Q, Lin W and Wang B: Regulation of ovarian cancer cell adhesion and invasion by chloride channels. Int J Gynecol Cancer 19: 526-530, 2009.

9. Kang MK and Kang SK: Pharmacologic blockade of chloride channel synergistically enhances apoptosis of chemotherapeutic drug-resistant cancer stem cells. Biochem Biophys Res Commun 373: 539-544, 2008.

10. Kunzelmann K: Ion channels and cancer. J Membr Biol 205: 159-173, 2005

11. Arcangeli A, Crociani O, Lastraioli E, Masi A, Pillozzi S and Becchetti A: Targeting ion channels in cancer: A novel frontier in antineoplastic therapy. Curr Med Chem 16: 66-93, 2009.
12. Conti M: Targeting ion channels for new strategies in cancer diagnosis and therapy. Curr Clin Pharmacol 2: 135-144, 2007

13. Guan YY, Wang GL and Zhou JG: The ClC-3 $\mathrm{Cl}^{-}$channel in cell volume regulation, proliferation and apoptosis in vascular smooth muscle cells. Trends Pharmacol Sci 27: 290-296, 2006.

14. Lemonnier L, Shuba Y, Crepin A, Roudbaraki M, Slomianny C, Mauroy B, Nilius B, Prevarskaya N and Skryma R: Bcl-2dependent modulation of swelling-activated $\mathrm{Cl}^{-}$current and CLC-3 expression in human prostate cancer epithelial cells. Cancer Res 64: 4841-4848, 2004.

15. Weylandt KH, Nebrig M, Jansen-Rosseck N, Amey JS, Carmena D, Wiedenmann B, Higgins CF and Sardini A: ClC-3 expression enhances etoposide resistance by increasing acidification of the late endocytic compartment. Mol Cancer Ther 6: 979-986, 2007.

16. Su J, Xu Y, Zhou L, Yu HM, Kang JS, Liu N, Quan CS and Sun LK: Suppression of chloride channel 3 expression facilitates sensitivity of human glioma U251 cells to cisplatin through concomitant inhibition of Akt and autophagy. Anat Rec 296: 595-603, 2013.

17. Xu Y, Zheng H, Kang JS, Zhang L, Su J, Li HY and Sun LK: 5-Nitro-2-(3-phenylpropylamino) benzoic acid induced drug resistance to cisplatin in human erythroleukemia cell lines. Anat Rec 294: 945-952, 2011.

18. Du S and Yang L: ClC-3 chloride channel modulates the proliferation and migration of osteosarcoma cells via AKT/GSK3 $\beta$ signaling pathway. Int J Clin Exp Pathol 8: 1622-1630, 2015.

19. Bruix J and Sherman M; Practice Guidelines Committee, American Association for the Study of Liver Diseases: Management of hepatocellular carcinoma. Hepatology 42: 1208-1236, 2005.

20. Bruix J and Sherman M; American Association for the Study of Liver Diseases: Management of hepatocellular carcinoma: An update. Hepatology 53: 1020-1022, 2011.

21. European Association For The Study Of The LiverEuropean Organisation For Research And Treatment Of Cancer: EASL-EORTC clinical practice guidelines: Management of hepatocellular carcinoma. J Hepatol 56: 908-943, 2012.

22. Chan SL, Mo FK, Johnson PJ, Hui EP, Ma BB, Ho WM, Lam KC, Chan AT, Mok TS and Yeo W: New utility of an old marker: Serial alpha-fetoprotein measurement in predicting radiologic response and survival of patients with hepatocellular carcinoma undergoing systemic chemotherapy. J Clin Oncol 27: 446-452, 2009.

23. Keam B, Oh DY, Lee SH, Kim DW, Im SA, Kim TY, Heo DS and Bang YJ: A phase II study of 5-fluorouracil and cisplatin systemic chemotherapy for inoperable hepatocellular carcinoma with $\alpha$ fetoprotein as a predictive and prognostic marker. Mol Med Rep 1: 415-422, 2008.

24. Ye D, Luo H, Lai Z, Zou L, Zhu L, Mao J, Jacob T, Ye W, Wang L and Chen L: ClC-3 Chloride Channel Proteins Regulate the Cell Cycle by Up-regulating cyclin D1-CDK4/6 through Suppressing p21/p27 Expression in Nasopharyngeal Carcinoma Cells. Sci Rep 6: 30276, 2016.

25. Wondergem R, Gong W, Monen SH, Dooley SN, Gonce JL, Conner TD, Houser M, Ecay TW and Ferslew KE: Blocking swelling-activated chloride current inhibits mouse liver cell proliferation. J Physiol 532: 661-672, 2001.

26. Ji X, Shen Y, Sun H and Gao X: A novel anti-alpha-fetoprotein single-chain variable fragment displays anti-tumor effects in HepG2 cells as a single agent or in combination with paclitaxel. Tumour Biol 37: 10085-10096, 2016.

27. Zhang L, He T, Cui H, Wang Y, Huang C and Han F: Effects of AFP gene silencing on apoptosis and proliferation of a hepatocellular carcinoma cell line. Discov Med 14: 115-124, 2012.

28. Yu WF, Zhao YL, Wang K and Dong MM: Inhibition of cell proliferation and arrest of cell cycle progression by blocking chloride channels in human laryngeal cancer cell line Hep-2. Neoplasma 56: 224-229, 2009.

29. Wang B, Xie J, He HY, Huang EW, Cao QH, Luo L, Liao YS and Guo Y: Suppression of CLC-3 chloride channel reduces the aggressiveness of glioma through inhibiting nuclear factor- $\kappa \mathrm{B}$ pathway. Oncotarget 8: 63788-63798, 2017. 